\title{
ATPase Effects on pro-Nutrients-mTOR Release Long Fatty Acids Chains Which Under Mitochondrial Phos- pholipase, Synthase, \& Synthetase Effects form Three ROR-alpha, beta, gamma Isoforms
}

Research Article

Volume 1 Issue 1- 2021

\author{
Author Details \\ Ashraf Marzouk El Tantawi* \\ Biomedical molecular studies, Egypt \\ *Corresponding author \\ Ashraf Marzouk El Tantawi, Biomedical molecular studies, Egypt
}

Article History

Received: April 24, 2021 Accepted: May 14, 2021 Published: June 01, 2021

\begin{abstract}
RORs isoforms are so active biological molecules in lipid metabolism and in fat biosynthesis, that strongly dependent on and regulated by OPA1 mitochondrial genes and its active mitochondrial enzymes where each of mitochondrial enzyme (phospholipase, synthase, and synthetase) is responsible for its own ROR isoform (phospholipase responsible for ROR-alpha synthesis, synthase responsible for ROR-beta synthesis, and synthetase responsible for ROR-gamma synthesis for acting and functioning the long fatty acids molecules "which produced from the effects of ATPase and COX enzyme on lipid molecules which accompanied and associated with absorbed nutrient molecules (pro-lipo-nutrient -mTOR molecules)", and then will follow its own pathway in fatty and amino acids biosynthesis, in active anti-inflammations biosynthesis, and then will follow its own functions in original cells proliferations.
\end{abstract}

Where, Lipid molecules+ATPase+COX enzymes $ר>$ long Fatty acids molecules +mitochondrial active phospholipase + ribosomal ATPase (in a control limit) ᄀ-> long Fatty acid-acyl-CoA phospholipase (which is ROR-alpha active isoforms), 7$\urcorner>>$ Fatty acyl-CoAsynthase which is ROR beta active genes, And $\neg \neg>>$ fatty acyl-CoA-synthetase (which is ROR-gama isoforms) then both ROR-alpha and ROR-gama +AMP $\neg>$ isopentanyl PP+ Leu (or Meth) amino acids $\neg>$ leu-pentapeptides (or Meth-pentapeptides) active molecules for activating enkephalin tissue in brain.

ATPase and COX enzymes are responsible for acting on lipid associated with nutrients-mTOR for producing long fatty acids molecules which will be affected by mitochondrial phospholipase, synthase and synthetase to form ROR-alpha, ROR-beta and ROR-gamma respectively.

The Purpose of this study: Understanding the main origin of the three RORs isoforms molecules and the main function of each isoforms in lipid metabolism and in fat biosynthesis, dependent on mitochondrial enzymes effects on the long fatty acids chains (which produced from the effects of ATPase and COX-2 on lipid molecules) for producing:

1)acyl-CoA phospholipase (ROR-alpha),

2) acyl-CoA synthase (ROR-beta),

3) acyl-CoA-synthetase (ROR-gamma).

Material: FOX forkhead genes, RAR-related orphan receptor A (ROR alpha), Mitochondrial OPA1 gene and their effective enzymes synthetase, Synthase, and Phospholipase Enzymes, pro-mTOR Protein conjugated with lipid molecules, Ser/Thr sequences (included in Pro-mTOR protein) Phosphorylation mechanism, Phosphatidylcholine, Acetylcholine, Phospholipases A2 (PLA2s), Phospholipid membranes, Autophagosome membrane, long acyl-CoA synthase (ROR beta), Long acyl-CoA synthetase (ROR gamma), Long acyl-CoA phospholipase (ROR alpha), Lysosomes, peroxisomes membranes granules, Thromboxane-A2, tumor necrosis, factor - alpha (TNF- $\alpha$ ) subunits, prostaglandins, Liver X Receptor (LXR), Retinoid X Receptor (RXR), Keratinocytes, Interferon-gamma (IFN-gamma), nerve growth factor. 


\section{Introduction}

The retinoid-related orphan receptors (RORs) alpha, beta, and gamma isoforms comprise one nuclear orphan receptors gene subfamily, where the effects of mitochondrial enzymes on ROR genes will differentiate the ROR genes structure, activities, and specificity of functions.

The first steps of the pathways of the synthesis of the three RORs isoforms structures molecules is started by the effects of ATPase and COX-2 enzymes on lipid-nutrients (pro-nutrients-mTOR) molecules for generating long fatty acids molecular chains, which will be affected by mitochondrial anabolic enzymes (synthetase, synthase and phospholipase) on their molecular chains to form three active RORs isoforms molecules (RORs alpha, beta, and gamma), that will have same molecular structures but differ only in their terminal amino acids, where active RORs isoforms belong to 1st DNA strand, that DNA-binding domain is highly conserved among RORs isoforms, that are carrying basic imp characteristics sequences able to moderate the received pro-nutrient-mTOR with its conjugated complexed lipid to a proper active carboxylated long fatty acyl-CoA enzymatic molecules for regulating cholesterol productions, for hormones biosynthesis, for regulating active subunits and genes, for regulating steroid metabolic process, cells proliferations, cGMP metabolic process, for autophagosome membrane formations, for reactivating autophagy, and for circadian regulations of genes expression... etc.

\section{Methods}

The FOX forkhead genes and RORs isoforms biosynthesis are strongly depending on mitochondrial anabolic active enzymes activities (phospholipase, synthase, synthetase), that RORs biosynthesis are having a strong regulations by mitochondrial OPA1 membrane functions which act on the long fatty acids molecules which produced from the effects of ATPase and Cox enzymes on lipid molecules for releasing the three enzymatic acyl-CoA active molecules for the cholesterol production, for TNF with thromboxane (alpha, beta \& gamma) subunits productions, for sestrin-Leu 1 biosynthesis, for prostaglandins synthesis (due to inflammation) which reflect the strengthen of ROR-beta with ROR-alpha activities, for S6K1 production, and for estrogen \& insulin growth and their ratio biosynthesis.

When lipid associated with absorbed nutrient molecules (pro-lipomTOR) will be firstly affect by ATPase and by Cox 2 for releasing long fatty acids molecules and will be directed to FOX forkhead and to ROR genes, where first the ATPase and COX-2 will act on lipid molecules for releasing long fatty acids molecules then the mitochondrial anabolic enzymes effects will act fatty acids molecules for releasing the acyl-CoA-phospholipase (ROR alpha isoforms), acyl-CoA-synthase (ROR-beta), and acyl-CoA synthetase (ROR-gama isoforms), where all RORs isoforms are having the same molecular structure but differ only in their terminals.

The decreasing in mitochondrial synthetase enzyme will reflect inhibition or decreasing in acyl-CoA-synthetase enzymatic molecules (ROR-gamma) synthesis which adopted for carbohydrate metabolic cycles and the ratio of pyrimidine in biological molecules, that can reflect decreasing in the pyrimidine synthesis from purines which will lead to decreasing in hormone biosynthesis, and sestrin biosynthesis, and decreasing in RORs isoforms stabilities and activities, which can reflect decreasing in FOX genes stabilities, and decreasing in Thymine kinases molecules activities which consequently lead to inhibition or decreasing in cholesterol biosynthesis, and in estrogen or insulin hormones biosynthesis pathways.

Long-fatty acyl-CoAs are produced due to the effects of mitochondrial anabolic enzymes on the long fatty acids molecules which produced from the effects of ATPase and mitochondrial COX-2 on the lipid molecules, which considered as necessary anabolic regulatory and intermediates molecules in lipid metabolic pathways and in the active kinases molecules resynthesis during the binding of Pro-nutrientsmTOR molecules.

Following the long fatty acids formation by the effects of ribosomal ATPase is the effect of mitochondrial OPA1 membrane activities for producing its necessary anabolic mitochondrial enzymes (phospholipase, synthase, and synthetase) for producing the three RORs isoforms active enzymatic molecules acyl-CoA-phospholipase (ROR-alpha), acyl-CoA synthase (ROR-beta), acyl-CoA synthetase (ROR gamma).

The RORs isoforms considered as three structures of RORs isoforms, each isoform bind to one of the anabolic mitochondrial enzymes (synthase, synthetase, and phospholipase enzymes) to form its own ROR-isoforms, and each of RORs isoforms has its own specific functions and pathological pathways but is contributing with others RORs-isoforms for running and completing their specific biosynthesis pathways during lipids metabolism.

Those RORs (the enzymatic acyl-CoA) isoforms molecules are having same molecular structures but differs only in their terminal amino acids, and are incorporated into acylated proteins and complex active fatty molecules like triacylglycerol, phospholipids, and cholesterol esters for activating the brain metabolic processes, for the liver metabolic activities, and for the strengthen of immune efficiency through TNFAlpha and TXA2 subunits productions by the acyl-CoA-synthase (ROR beta isoforms) and for sestrin-Leu biosynthesis through the regulation of acyl-CoA-synthetase (ROR gamma isoforms).

The synthesis of each of acyl-CoA isoform firstly by the effects of ATPase and COX-2 on lipid molecules followed by the mitochondrial OPA1 activities for releasing its active enzymatic acyl-CoA isoforms during lipid metabolism.

As ribosomal ATPase and Cox enzymes act on lipo-mTOR protein as will release long fatty acids molecules which will be followed the mitochondrial effects on the long Fatty molecules for producing enzymatic acyl-CoAs (RORs) isoforms.

Where acyl-CoA isoforms molecules will be binded to whether synthetase, phospholipase, or to synthases enzymes as intermediates for brain activities, for liver functions... etc depending on the type of signals activities received from brain and from cells to form RORs isoforms.

The mitochondrial enzymes effects are so necessary (anabolic antiinflammatory enzymes) for RORs isoforms biosynthesis, and are considered as the main anti-inflammatory regulating enzymes tools, that phospholipase, synthetase, and synthase and are so necessary for TNF-beta and for thromboxane-beta (TXA2) biosynthesis, and regulate the releasing of the active four kind of kinases proteins during the FOX binding activities, and act as main mediators for anti inflammations pathways as for prostaglandins productions, and are acting for releasing the ROR-alpha isoforms (by phospholipase) which are active isoforms for phospholipid membranes and for antigen biosynthesis.

Liver X receptors (LXRs) are nuclear receptors, that LXRs were originally considered as 'orphan' nuclear receptors, and it's origin depend on the synthesis of acyl-CoA phospholipase isoforms which is the basic of liver cells phospho-lipo-membrane. however, those receptors were 'adopted' for running lipid metabolism, cholesterol synthesis, and phospholipid membranes which act as so imp biological filter for and from nucleus.

The phospholipid membranes formed from acyl-CoA-phospholipase (ROR-alpha) isoforms functions, where phospholipid are imp 
molecules for skeletal muscle development, for photoreceptor development and for liver activities.

IFN gamma depending on biosynthesis effects of synthetase enzymes on long fatty acids chains for producing fatty acyl-CoAs synthetase which act as a protective IFN-gamma in regulating the retinal hydrations.

PLC-gammal is implicated in a variety of cellular signaling and processes including mitogenesis and calcium entry.

The Nerve growth factor is a neurotrophic factor and neuropeptide primarily involved in the regulation of growth, maintenance, and proliferation that has imp roles in carbohydrate biosynthesis for maintaining the necessary balances of purines related to pyrimidine nucleotides through the pyrimidines synthesis where their results from the effects on carbohydrate will feed the sensor nerve and neuronal hyper reactivity for nerve activities through the mediation of the effects of synthase enzymes for beta subunits productions.

The functions of the effects of synthase on long fatty acids is the productions of ROR-beta then followed by the effects phospholipase for ROR-alpha isoforms synthesis and productions for cells proliferations and their biosynthesis pathways, where are cooperating together for running so imp osteogenic repressor in regulating bone formation and in new blood cells (rounded circular cells) synthesis with their effective phospholipid membranes. The synthase mitochondrial enzymes are so imp for TNF-beta, Thromboxane-beta subunits productions which will be followed by the effects of phospholipase for TNF-alpha and for TXA2-alpha linear subunits productions, and for circular rounded anti-inflammatory genes (as autophagy synthesis), where PSTC-kinases (mTORC1) and thromboxane-A2 are so imp for the autophagy reactivities and biosynthesis.

Heart failure is associated with decreased myocardial fatty acid metabolic pathways and decreasing in fatty acids functioning and oxidation capacity and has been likened to energy starvation. As the adenosine metabolism in tissues have been consumed due to extra ATPase and COX-2 activities as energy utilization will increased from breaking and analyzing genes and fatty acyl-CoA molecules and from pyrimidine molecules lead to precipitation of ub normal molecules which can block blood fluidity in arteries and veins leads to decreasing in heart muscle lead to heart failure.

Active RORs isoforms belong to 1st DNA strand where, DNA-binding domain is highly conserved among RORs isoforms, that are carrying basic imp characteristics sequences able to moderate the received pronutrient-mTOR with its conjugated complexed lipid to a proper active carboxylated long fatty acyl-CoA enzymatic molecules for regulating cholesterol productions, for hormones biosynthesis, for regulating active subunits and genes, for regulating steroid metabolic process, cells proliferations, cGMP metabolic process, for autophagosome membrane formations, for reactivating autophagy, and for circadian regulations of genes expression... etc.

Lipid metabolism start by the effects of ATPase and COX-2 on the pro-nutrients-mTOR molecules for releasing long fatty acids molecules directed to FOX forkhead genes for binding with the effects of mitochondrial OPA1 functions on fatty acids molecular chains, where lipid will be separated by effect of mitochondrial enzymes (phospholipase, synthase, synthetase) through OPA1 genes functions for the enzymatic acyl-CoA-"phospholipase, synthase, or synthetase" isoforms for fat, and for lipoproteins biosynthesis.

The biosynthesis of acyl-CoA phospholipase ROR alpha, acyl-CoAsynthase the ROR-beta, acyl-CoA synthetase ROR-gamma, are strongly depending on the mitochondrial enzymes effects on fatty molecules, but firstly depends on the effects of ATPase and Cox2 enzymes on the lipid conjugated to mTOR" molecules.
The synthesis of each of acyl-CoA isoforms are by mitochondrial effects on fatty molecules for releasing the three enzymatic acylCoA isoforms in the lipid metabolic pathways, in the pathogenesis of metabolic disease and carcinogenesis [1].

Increasing in fatty acid results in an induction of genes promoting fatty acid oxidation [2]. Where, increasing in fatty acids will stimulate mitochondrial effects for releasing its enzymes for the three enzymatic acyl-CoA isoforms synthesis for cholesterol production which is the substrate for estrogen and insulin during their synthesis.

The increasing in the enzymatic fatty acyl-CoAs by mitochondrial effects will increase. Inductions of genes by promoting fatty acid oxidation, but in case of deficiency in the OPA1 activity the ATPase and COX-2 catabolic processes on analyzing lipid molecules will increase for releasing irregular results of oxidative fatty molecules which will be the basic of irregular subunits synthesis the will be the main reason for fat deposits in the arteries walls, and the reason for the symptoms of atherosclerosis and heart disease.

The decreasing in long fatty acids chains productions and in ROR isoforms synthesis, will lead to increasing in complexed irregular fatty molecules availabilities for energy utilization and for catabolic processes that will reflect inhibition in pyrimidine synthesis and inhibitions in beta and alpha oxidative processes, that will lead to inhibition in normal cholesterol synthesis that will lead to IGF-I productions with inhibition in the proper original estrogen (dépend on type of inhibitions) and in normal insulin production, lead to precipitation of fatty acid irregular lipo-molecules in capillaries and blood vessels lead to Arteriosclerosis, cardiovascular diseases, and osteoarthritis.

Where, Lipid molecules+ATPase $ר>$ long Fatty acids molecules + mitochondrial active phospholipase + ribosomal ATPase (in a control limit) ᄀ-> long Fatty acid-acyl-CoA phospholipase (which is ROR-alpha active isoforms), ר>>Fatty acyl-CoA-synthase which is ROR beta active genes, And $\neg \neg>>$ fatty acyl-CoA-synthetase (which is ROR-gamma isoforms) then both ROR-alpha and RORgamma +AMP $\neg>$ isopentenyl PP+ Leu (or Meth) amino acids $\neg>$ leupentapeptides (or Meth-pentapeptides) active molecules for activating enkephalin tissue in brain.

The decreasing or inhibition of synthases, synthetase or in phospholipase enzymes for releasing RORs active isoforms during Pro- Nutrients-mTOR metabolic pathways will reflect the increasing in random irregular oxidative fatty acids due to oxidative processes by ATPase and by COX-2 enzyme with inhibition in enzymatic acyl-CoA isoforms productions lead to several precipitations of unknown lipomolecules and fatty acids in capillaries that will block blood follow in blood vessels and will inhibit other dependent pathways steps lead to decreasing in muscles activities and heart functions.

That acetylation of the fatty acid $\beta$-oxidative enzymes, and in $\beta$-HAD ( $\beta$-hydroxyacyl-CoA dehydrogenase) is associated with an increase in activity and fatty acid oxidation in heart from obese mice with heart failure [3] where, increasing of acetylation of beta hydroxy-acyl-CoA which is the main component of mitochondrial matrix and originally formed by feedbacks of RORs isoforms, will lead to increasing in +ve gps in B-HAD molecules that will alter the BHAD active sites in mitochondrial matrix, that will lead to reductions or inhibition in mitochondrial filtration (where fatty acids need to pass through mitochondrial membrane to reach matrix for B kit-acyl-CoA synthesis during pro-nutrients-mTOR metabolic pathways) that will lead to reductions or inhibition in releasing the mitochondrial enzymes which will lead to reduction or inhibition in releasing the RORs isoforms, reduction or inhibition in lipid and protein metabolism, and reductions or inhibition in regular cholesterol biosynthesis and consequently will lead to reduction or inhibition in estrogen and in insulin biosynthesis. 
3-Hydroxy-3-methylglutaryl-COA (HMG CoA) reductase produces mevalonate, an important intermediate in the synthesis of cholesterol and essential nonsterol isoprenoids [4]. Where, 3-Hydroxy-3methylglutaryl-coenzyme A (HMG-CoA) reductase is the product of mitochondrial matrix through the passages of fatty acids molecules through mitochondrial membrane to reach the BHAD that synthase enzymes will act on fatty acids molecules for releasing the RORsynthase isoforms (ROR-beta) and HMG-CoA which intermediate the mevalonate molecules productions which intermediate the cholesterol synthesis.

Where mevalonate also is an intermediate molecules for reactivating brain acetylcholine and enkephalin tissue for both Meth and leupentapeptides biosynthesis in enkephalin in brain tissue.

The BHAD and HMG-CoA are both intermediate molecules through the activity of acyl-CoA-synthase (ROR-beta isoforms) for mevalonate productions, for estrogen and normal insulin biosynthesis.

Protagonists 1 PGs, are a family of fatty acid eicosanoids synthesized from arachidonic acid via cyclooxygenase enzymes [5]

That Rho proteins due to effects of ATPase and COX-2 on phospholipid membranes and are involved in the expression of proinflammatory cytokines, Rac proteins modulate reactive oxygen species (ROS), whereas Ras protein are synthesized from acyl-CoAphospholipase (ROR-gamma isoforms) functions and are involved in cell proliferation, and cell adhesion [6].

Due to cells death the phospholipid membranes will be break and analyzed by ATPase and COX-2 enzymes effects for releasing cyclooxygenase enzyme that will Determine and characterized its molecular structure and functions.

Phospholipid membrane are mainly formed by the function of long fatty acyl-CoA-phospholipase (upon the effects of mitochondrial phospholipase enzymes on long fatty acids chains) which directed for phospholipid membrane biosynthesis.

As Pro-nutrients-mTOR molecules directed to FOX forkhead genes and to ROR genes with the regulation of mitochondrial enzymes effects, as the FOX genes will begin for releasing the four types of kinases proteins and the three RORs isoforms will be synthesis from fatty acids molecules molecules through the effects of mitochondrial effects (that fatty acids molecules formula by effects of ATPase and COX-2 on lipid molecules for generating long fatty acids chains) on the long fatty acids molecules for generating the three enzymatic acylCoA molecules "RORs isoforms" for cholesterol synthesis and for estrogen and insulin hormones biosynthesis.

The most necessary mitochondrial enzymes in lipid metabolism is the phospholipase enzyme, which responsible for releasing acylCoA-phospholipase (ROR-alpha isoforms) which is necessary for the formation the cell phospho-lipid membranes, for cells antigen synthesis, for cells proliferations, and for brain cells biosynthesis.

The Pro-nutrients-mTOR molecules + ATPase +COX-2 ר>>long Fatty acids molecules ++ mitochondrial enzymes effects $\neg>\neg>1$ ) acyl-CoA phospholipase molecules (ROR-alpha isoforms), 2) acylCoA synthase molecules (ROR-beta), and 3) acyl-CoA synthetase molecules (ROR gama isoforms), where those enzymatic fatty acylCoA proteins molecules (RORs isoforms) are having same molecular structure but differ only in their terminal amino acids, and each has its own activity and Participate in the other two RORs isoforms activities.

RORs isoforms activities are giving the soft, flexibility, and malleable characters to synthesis protein and genes that can help living cells to gains the persistence to high-temperature and low-temperature characteristics during their cellular metabolism.
The RORs isoforms are formed through The effects of mitochondrial enzymes synthetase, synthase and phospholipase on long fatty molecules (which produced from the effects of ATPase and some cases with mitochondrial COX-2 enzymes) for producing 1,acylCoA synthase 2,acyl-CoA synthetase And 3, acyl-CoA phospholipase (Figure 1) which have the function of lipoprotein synthesis, antigen synthesis, cells phospholipid-membrane synthesis, and TNF-a, TXA2 synthesis... etc. Where, all RORs molecules have same molecular structure and differ only on their terminal amino acids.

liver has two major sources of blood. The portal vein brings in bloodrich-nutrients from the digestive system, and the hepatic artery carries oxygenated blood and metabolized genes and protein. Bile productions by liver helps lipid metabolism and metabolizing some vitamins, that bile consists of bile salts, cholesterol, bilirubin and which are needed for metabolizing fats molecules then for estrogen and insulin biosynthesis.

Metabolizing carbohydrates within liver, are needed for maintain normal glucose levels, where that process is carried and done by acyl-CoA-synthetase functions (gamma isoforms) where the main function of carbohydrate processes is converting purines to necessary pyrimidines for rebuilding necessary hydrophobic amino acids for others two RORs isoforms processes and for Sestrins-Leu synthesis which is necessary for liver activities.

Then the results from the effects of acyl-CoA synthetase ROR gamma on carbohydrate is the glycogen which stored and released whenever a quick burst of energy is needed. The liver store vitamins A, D, E, K, and B12 which will be metabolized for RORs isoforms biosynthesis and hormones synthesis. Liver stores iron from hemoglobin in the form of ferritin, ready to make new red blood cells through the acylCoA-phospholipase alpha isoforms activities within liver too (Figure 2).

Liver adopted for interstitium fluid synthesis which carried out by hepatic arteries, and the liver has a major function which is filtering blood from inflammations, from irregular oxidized molecules... etc. Liver contains high numbers of Kupffer cells that are involved in immune activity, where the metabolizing proteins and Filtering the blood within liver need the activity of beta isoforms acyl-CoAsynthase for TNF-a and TXA2, water production which will help the processes which need to be done by others two RORs isoforms.

Liver X receptors (LXRs) are nuclear receptors, that LXRs were originally considered as 'orphan' nuclear receptors, and it's origin depend on the synthesis of acyl-CoA phospholipase isoforms which is the basic of liver cells phospho-lipo-membrane. however, those receptors were 'adopted' for running lipid metabolism, cholesterol synthesis, and phospholipid membranes which act as as so imp biological filter for and from nucleus.

The phospholipid membranes formed from acyl-CoA-phospholipase (ROR-alpha) isoforms functions, where phospholipid are imp molecules for skeletal muscle development, for photoreceptor development and for liver activities. The heterodimer binding to LXRresponsive elements (LXREs) in DNA consists of direct repeats (DRs) of the core sequence AGGTCA \} separated by 4 nucleotides (DR-4) [7].

The direct repeats of binding with DNA is Arg, Ser (where Ser is the so necessary preserved amino acids for acyl-CoA phospholipase, synthase, and synthetase in liver $\mathrm{X}$ receptor activities where Liver $\mathrm{X}$ receptor during lipid metabolism and for kind of "PSTk" protein kinases productions through FOX binding mechanism. And it has been Revealed that liver X Receptor-Retinoid X Receptor (LXRRXR) Heterodimer Cistrome Reveals Coordination of LXR and AP1 Signaling in Keratinocytes [8]. LXRs activate lipid metabolism 
through the production of polyunsaturated fatty acids (PUFAs) and their distribution into phospholipids via the control of FA desaturases, FA elongates, Lys phosphatidylcholine acyltransferase (LPCAT3), and phospholipid transfer protein (PLTP) [9]. The LXRs and retinoids $\mathrm{X}$ receptor (RXR) are coordinate for phospholipids biosynthesis through the acyl-CoA phospholipase activity on the catalyzing the transacylation reaction between phosphotidylcoline (LPC) molecules for generating diacylphospholipid (Figure 3).

Also, fatty acyl-CoA phospholipase isoforms (ROR gamma) has imp roles in reactivating brain cells through the biological transacylations between phosphotidylcoline by acyl-CoA phospholipase isoforms for generating diacylphospholipid which reactivate enkephalin cells. The negative feedback regulation of cholesterol metabolism in mammalian cells ensures the responsibility and functions of ROR-alpha genes and the polyunsaturated fatty acids (PUFAs) molecules production in cholesterol biosynthesis, and the function of ROR-alpha isoforms for phospholipids synthesis and their distributions for ensuring nucleus activities.

That it has been approved the negative feedback regulation of cholesterol biosynthesis in mammalian cells ensure the proper balance of cholesterol with other membrane lipids, principal among these being the major phospholipid phosphatidylcholine (PC) [10]. Indicating that fatty acyl-CoA-phospholipase which have the function of phospholipid membranes biosynthesis are having the same function of cholesterol biosynthesis where both cholesterol and phospholipids membranes are having proper balance in lipid metabolism and in lipoprotein biosynthesis, and indicating that the fatty acyl-CoAphospholip isoforms are having the function of activating brain functions through phospholipid phosphatidylcholine biosynthesis and consequently by reactivating the enkephalin pentapeptides (Meth and Leu) in brain.

The Polar Head Modified Phospholipids by Phospholipase D-Catalyzed Transformations of Natural Phosphatidylcholine [11]. Phosphatidylcholine (PC) synthesis is necessary for remodeling of exogenous phospholipids, necessary for prostaglandins synthesis during anti-inflammatory processes, and for autophagosome membrane. That phosphatidylcholine (PC) synthesis is required for autophagosome membrane formation and maintenance during autophagy [12].

Phospholipases A2 (PLA2s) are essential enzymes in cells, where are not only responsible for maintaining the structural organization of cell membranes, but also play a pivotal role in the contributions of the regulations of cells functions through its contributions to the others active two RORs beta and gamma isoforms.

That the acyl-CoA phospholipase are playing imp roles during inflammation that catalyze the transacylation of phosphatidylcholine for phospholipid membranes synthesis. The Lysophospholipase catalyzes the hydrolysis of the carboxyl ester bonds of lysophospholipids. Some lysophospholipase isoforms are known to catalyze a transacylation reaction between two lysophosphacholine LPC molecules, leading to the formation of a diacylphospholipid PC and GPC [13] (Figure 4).

Interferon-gamma (IFN-gamma) is formed from the phospholipase effects on long fatty acids molecules for releasing acyl-CoA synthetase ROR-gamma) isoforms, where, IFN-gamma receptors are mainly localized to the basolateral membrane of human retinal pigment epithelium and has a protective roles in retinal hydrations across the outer blood-retinal barrier in inflammatory disease, and provide the basis for possible therapeutic interventions [14].

During IFN intervention to any foreign molecules related to cells, will act by analyzing and break down those molecules and convert some of its components into acceptable units by living cell throughout the mechanism of converting purines into pyrimidines, which contribute the possibility of implementing Beta-oxidative processes to produce modified TNF-a and Thromboxane-A units (upon the inflammation stimulators factors), that previous mechanism includes hydration production through cells metabolic processes. IFN gamma depending on biosynthesis effects of synthetase enzymes on long fatty acids chains for producing fatty acyl-CoAs synthetase which act as a protective IFN-gamma in regulating the retinal hydrations.

PLC-gamma1 is implicated in a variety of cellular signaling and processes including mitogenesis and calcium entry. The Nerve growth factor is a neurotrophic factor and neuropeptide primarily involved in the regulation of growth, maintenance, and proliferation that has imp roles in carbohydrate biosynthesis for maintaining the necessary balances of purines related to pyrimidine nucleotides through the pyrimidines synthesis where their results will feed the sensor nerve and neuronal hyper reactivity for nerve activities.

The phospholipase activity of PLC-gammal plays an essential role in nerve growth factor (NGF)-triggered Raf/MEK/MAPK pathway activation in PC12 cells [15]. The PLC-gamma1 is the phospholipasealpha (PLC-alpha) associated to and with ROR-gamma activities for the contributions of the synthesis of nerve growth factor neuropeptides activities which need the fatty acyl-CoA synthetase acting on carbohydrate for pyrimidines synthesis which needed for hydrophobic amino and fatty acids synthesis with the contributions of fatty acyl-CoA phospholipase isoforms for neuropeptides synthesis.

The ROR gamma isoforms molecule is the effect of synthetase on long fatty acids molecules to form long fatty acyl-CoAs synthetase which act on carbohydrate molecules (which is necessary for regulating the pyrimidine synthesis from purines molecules) for TNF-a and TXA2 subunits productions and hydration productions for assisting the others two RORs isoforms activities.

Each of those RORs isoforms structures will be synthesis through the effects of mitochondrial enzymes on long fatty molecules to generate long enzymatic acyl-CoA isoforms where each has its own specificity with its contributions to the other two RORs isoforms (where all three RORs isoforms are strongly and closely related to each other) for phospholipid synthesis and to give the vitality to tissues through fat, proteins, and carbohydrate biosynthesis pathways.

Citrate Synthase (CS) is a direct effect of ROR-beta isoforms on long fatty molecules during fat biosynthesis by which ROR-beta regulates carbohydrate and fat biosynthesis via regulation of CS expression. CS plays a critical role in providing citrate derive acetyl-CoA for lipogenesis and cholesterol genesis [16].

The retinoid-related orphan receptor gamma-t (ROR $\gamma \mathrm{t})$ did not generate Th cells with robust IL-17 and IFN- $\gamma$ expression [17]. Where, the robust IL-17 and IFN- $\gamma$ in tissue reflect the robust acyl-CoA synthetase reflect enough robust ROR-gamma isoforms for acting on inflammations without need for more T-helper cells reproductions.

the effects of synthase and phospholipase on the productions of RORs beta and alpha isoforms respectively are cooperating together for running so imp osteogenic repressor in regulating bone formation and in new blood cells synthesis with their effective phospholipid membranes, and are imp for TNF-a and TXA2 alpha subunits productions, where PSTC-kinases (mTORC1) and thromboxane-A2 can used for the autophagy reactivities and biosynthesis.

The ROR-beta (ROR- $\beta$ ) isoforms, act as a member of the orphan nuclear receptor family which plays so important regulatory role in the maintenance of a variety of physiological and pathological processes under the stimulations of the inflammatory factors, where the effect of synthase on long fatty acids molecules is considered as a maintenance 
steps which release TNF-a and TXA2 during pathological processes. $\operatorname{ROR} \beta$ has been determined to act as an osteogenic repressor in regulating bone formation and is involved in regulating circadian rhythm [18]

The ROR alpha isoforms molecule is the effect of phospholipase enzymes on long fatty acids chains to generate ROR-alpha genes molecules, the ROR beta is the effect of synthase on ROR gene to generate ROR-beta genes molecules, but ROR-gamma molecule is the effect of mitochondrial synthetase enzymes on ROR gene to generate ROR-gamma gene molecules.

During inflammation effect the acyl-CoA synthase and acyl-CoAsynthetases of (ROR gamma) will be released to act on inflammations molecules to ensure converting some of purines (which involved in inflammations) to pyrimidine nucleotides for rebuilding necessary hydrophobic amino acids then will be followed by acyl-CoA-synthase for TNF and TXA2 synthesis. Where synthase (CS) plays a critical role in providing citrate derived acetyl-CoA for lipogenesis and cholesterol genesis [19], that the mitochondrial synthase enzyme is necessary for the reactivating ROR-beta isoforms and for FOX genes activities through PSTTK, PSTGK and PSTCk synthesis during the pro-mTOR pathways for phospho-lipo-protein and cholesterol biosynthesis and for estrogen and insulin biosynthesis.

Peroxisomes, are like lysosomes are enclosed by a single membrane. Peroxisomes are resemble lysosomes in being filled with enzymes. However, peroxisomes bud off from the endoplasmic reticulum, not the Golgi (which package proteins and lipid molecules, especially proteins destined to be exported from the cell) as lysosome.

Peroxisomes is organelles for manufacturing cell needs in favor of inner cells components such as phospholipid membranes, lipoproteins, and production of TNF-a and TXA2 subunits which can be Headed later to lysosomes for T-cells and autophagy reactivations.

Peroxisomes are indispensable for proper functioning of human cells. They efficiently compartmentalize enzymes responsible for a number of metabolic processes, including the absolutely essential beta-oxidation of specific fatty acid chains. These and other oxidative reactions produce hydrogen peroxide, which is, in most instances, immediately processed in situ to water and oxygen [20].

As phospholipase enzyme in peroxisomes decreased or inhibited will lead to accumulation of hydrogen peroxide and downstream of reactive oxygen species including beta oxidative processes and alpha oxidative processes in the heme-containing tetrameric enzyme, catalase. Peroxisomes are ubiquitous organelles which participate in a variety of essential biosynthesis pathways including lipid metabolism and fatty acids biosynthesis.

An intimate interrelationship between peroxisomes and mitochondria is emerging in mammals by effects of mitochondrial enzymes on long fatty acids chains for producing the three enzymatic active RORs isoforms which I consider that the three acyl-CoA RORs isoforms are stored in the forms of peroxisomes for rapid effects in anti-inflammations in fat biosynthesis including phospholipids synthesis, in hydrophobic fatty acids and amino acids synthesis, and in carbohydrate biosynthesis, where both organelles cooperate in fatty acid $\beta$-oxidation and cellular lipid homeostasis. Acyl-CoA dehydrogenases (ACADs) are bona fide peroxisomal proteins in fungi and mammals and together with acyl-CoA oxidases (ACOX) belong to the basic enzymatic repertoire of peroxisomes [21].

Peroxisomal organelles are made basically by mitochondrial effect on fatty acids due to RORs genes transcriptional filter and consist of the three RORs isoforms receptors alpha, beta, \& gamma) for easier fastest processes for alpha beta, and gamma oxidations.
The beta oxidative process occur due to the activity of synthase for running the TNF-a and TXA2 biosynthesis for increasing immune efficiency, alpha oxidative processes is the fatty acyl-CoA phospholipase activities for lipoprotein and phospholipid biosynthesis where that alpha and beta oxidations are linked and contributing together for running their activities and producing $\mathrm{H} 2 \mathrm{O}$ from the peroxisomal processes, but gamma oxidative processes are linked to type of nutrients mTOR molecules, and to availabilities of Ser amino acids for releasing Thymine kinases proteins for the acyl-CoA synthetase biosynthesis, and linked to Thymine pyrimidine and production from purines nucleotides during carbohydrate metabolism.

At the time of decreasing or inhibition in acyl-CoA phospholipase and in gamma acyl-CoA synthetase will lead to accumulation of hydrogen peroxide and downstream of reactive oxygen species.

Specific peroxisomal acyl-CoA synthetase (ACS) participate in peroxisomal pathways in lipid metabolism [22]. Peroxisomal ACSs has its imp roles in maintaining the proper balance of purines related to pyrimidines that can be done through its functions in carbohydrate biosynthesis, and that ACSs (ROR gamma) have overlapped responsibilities with the others alpha and beta isoforms, because these enzymes are so depending on each others and play critical roles through their contributions together for the proper cellular fat + carbohydrate + proteins biosynthesis.

ROR) $\beta$, act as a member of the orphan nuclear receptor family, plays an important regulatory role in the maintenance of a variety of physiological and pathological processes. ROR $\beta$ has been determined to act as an osteogenic repressor in regulating bone formation, and is involved in regulating circadian rhythm [23].

Beta oxidative biosynthesis are processes that the synthase enzymes (conjugated to fatty acids molecules whether fatty acetyl CoA or fatty acyl-CoA) are acting on inflammations molecules and on nutrientsmTOR for producing TXA2 and TNF-a subunits.

Which are involved in bone formation and are considered as prostanoids by some scientists. Where, prostanoids synthesis profile is dependent on differences in enzymatic expression in cells under inflammation factors, and specifically depend on the regulations of synthase enzymes which regulate TNF-a and Thromboxane-A productions under the stimulations of inflammations. Macrophages, for instance, release prostaglandins PGE2 and TXA2 [24].

Where during inflammation the effects of synthase enzymes as the ROR beta isoforms on inflammations and on irregular nutrients mTOR molecules for producing TNF-a and TXA2, and in the main time phospholipase as in the ROR-alpha isoforms will act on the same inflammation molecules for releasing prostaglandins (if possible) where both RORs isoforms playing so imp roles in osteogenic repressor in regulating bone formation with the new blood cells production with their effective phospholipid membranes.

It has been reported that IFN-beta-1a treatment down-regulated the expression of IL-1beta in DCs. We propose that IFN-beta-1a-mediated up-regulation of the suppressor of cytokine signaling 3 expression, induced via STAT3 phosphorylation, mediates IL-1beta and IL-23 down-regulation, while IFN-beta-1a-induced STAT1 phosphorylation induces IL-27p28 expression [25].

IFN gamma depending on the biosynthesis effects of synthetase enzymes on long fatty acids chains for producing fatty acyl-CoAs synthetase which contribute for the IFN-acyl-CoA synthase biosynthesis (IFN beta) production which depends on long fatty acyl-CoA-synthase biosynthesis from the contribution of IFN-gamma which will be responsible for STAT3 phosphorylated protein biosynthesis which intermediate for the beta IL27 p28 synthesis. Where IFN gamma is 
acting on fat and carbohydrate molecules biosynthesis for generating hydrophobic fatty and amino acids (and branched active fatty acids depending on the number of condensative fatty acyl-CoA molecules) and maintaining the proper balance of pyrimidine nucleotides formed from purines nucleotides in inflammatory molecules for the hydrophobic amino and fatty acids biosynthesis which used for the stat3 phosphorylated protein productions, which later responsible for intermediate for IL-27P28 biosynthesis. Also IFN-gamma act protective molecules in regulating the retinal hydration through its roles on fatty acids and on carbohydrate molecules in maintaining the proper balance of pyrimidine from purines in tissue that inducing a proper protective hydrations for retinal tissue.

functionality. Transforming growth factor- $\beta 1$ (TGF- $\beta 1$ ) is a pivotal mediator involved in airway remodeling that correlates with enhanced TH17 activity in individuals with severe asthma and is essential to TH17 differentiation and IL-17A production. IL-17A can also reciprocally enhance activation of TGF- $\beta 1$ signaling pathways, whereas combined TH1/TH17 or TH2/TH17 immune responses may additively impact asthma severity [26].

TGF- $\beta 1$ is originally regulated by IFN -gamma and beta which are reciprocally enhancing for the activation of TGF- $\beta 1$ signaling biosynthesis combined to the TH1/TH17 or TH2/TH17 immune responses, for re-enhancing the TH17 activities in individuals with severe asthma. where the proper reactivating TGF- $\beta 1$ from IFN-gamma then from IFN-beta will include the TNF-a and Thromboxane-A2 of the resynthesis for rebuilding and strengthen immune efficiency.

Protection from hepatocellular damage correlated with decreased levels of hepatic T-cell and macrophage infiltration and decreased expression of inflammatory mediators associated with NAFLD. In sum, our results indicate that the IL-17 axis also plays a role in a MCDD-induced model of NAFLD pathogenesis [27].

IFN gamma depending on the biosynthesis effects of synthetase enzymes will contribute for the IFN-acyl-CoA synthase biosynthesis (IFN beta) production which intermediate for STAT3 phosphorylated protein production which intermediate for TGF- $\beta 1$ production and consequently for T Helper cells and IL17A productions.

That at the time of decreasing or inhibitions in TNF-gamma synthesis and activities will affect on TNF beta structure and functions that will lead to dysfunction in T Helper cells molecular structure and activities that will lead to releasing un proper mutated IL17A that will help in increasing in inflammations, but the proper normal IL17A originally regulated from proper normal IFN-gamma for generating proper normal IFN beta will show decreasing in inflammation and protection to hepatic cells.

Decreasing or dysfunction in acyl-CoAs-synthetase will lead to decreasing on or dysfunction in acting on inflammations molecules and decreasing in pyrimidine synthesis from purines lead to increasing in purines, and decreasing in carbohydrate biosynthesis and increasing in mutated acyl-CoA-synthase and acyl-CoA-phospholipase and increasing in undigested fatty acids with decreasing in hydrophilic fatty and amino acids, lead to Fat deposits and atherosclerosis.

Where Those deposits may are made up of cholesterol, or by fatty acids molecules with cellular waste products, calcium and fibrin (a clotting material in the blood). As plaque builds up, the wall of the blood vessel thickens.

There are many plausible mechanisms by which an increase in adipose tissue could adversely affect the vessel wall but mainly due to decreasing in ROR-gamma isoforms activities. These include the changes in blood pressure, including in glucose (purines) level, decreasing in lipid/lipoprotein metabolism (mainly decreasing in fat biosynthesis), and systemic inflammation. by the decreasing in acyl-CoA synthetase
(ROR-gamma) will influence the function of endothelial cells, arterial smooth muscle cells, and macrophages in the vessel walls.

Using purines as a marker in macro vascular disease better than Using the measurement of coronary artery calcium as a marker for coronary atherosclerosis, which will indicate the decreasing in pyrimidine synthesis and the decreasing in fat biosynthesis.

If the normal cholesterol found deposits on vessel walls so there are a deficiency in acyl-CoA-phospholipase functions. But if deposits is contained mutated insulin-like (IGF-I) that indicate deficiency in normal cholesterol synthesis, with increasing in the mutated insulin like IGF-I molecules synthesis, which will reflect the lacking of hydrophobic (fatty and amino) acids synthesis from pyrimidine which originally formed from purines nucleotides by ROR-gamma isoforms activities

ROR-gamma is the main 1st active process in RORs isoforms biological mechanism, where ROR-gamma will actually feed ROR-beta for performing its necessary beta oxidations for producing IFN-beta-1amediated up-regulation of the STAT3 phosphorylation productions which necessary for IL-27p28.

IFN gamma depending on the biosynthesis effects of synthetase enzymes on long fatty acids chains for ROR-gamma synthesis for the contributions of the ROR-beta synthesis for performing its necessary beta oxidations for strengthen immune, then for contributing the ROR-alpha (acyl-CoA phospholipase) synthesis for phospholipid synthesis during cells proliferations.

The synthesis of hydrophobic amino and fatty acids is depending mainly on pyrimidine synthesis from purines during carbohydrate and fatty biosynthesis by ROR-gamma activities is the main basic for the beta-oxidative synthesis processes for reactivating autophagy including their cytokines, then both ROR-gamma and ROR-beta are so necessary for ROR-alpha synthesis and functions respectively. At the point of dysfunction in ROR-alpha will reflect decreasing in phospholipid membranes synthesis that will reflect decreasing in cells membranes and cells proliferations synthesis that will lead to.

The cytokines and the microwave swim in the cytoplasm fluently in the straight long strands, not twisted, which are ready to involve in any metabolic cycles and attack any foreign body and drift in metabolic cycles, without protection by being in the twist form of cell structure that contains its internal components in a twisted forms and wrapped in protected structures by phospholipid membranes. The long, coiled form of the gene depends on the presence of serine and methionine in the straight genes filaments.

Vascular endothelial growth factor (VEGF) activates unfolded straight linear protein response sensors in the endoplasmic reticulum through phospholipase C gamma which mediated crosstalk with mammalian target of rapamycin complex 1 (mTORC1) in the regulation of angiogenesis [28]. Indicates the involvement of the synthesis of VEGF-A during the effects of phospholipase and mitochondrial enzymes on Pro-TOR protein during the binding with FOX transcription factor for re-synthesis pyrimidine and leucine amino acids for preparation for sestrin-1 synthesis. After pro-TOR protein which response to nutrition's, it will contain sugar (purines nucleotides) and branched fatty acids (BFA) molecules which their physical molecular structure will give signals for stimulating the ribosomes and the mitochondrial activities to excrete their necessary enzymes for acting on the extra purines and on branched fatty acids (BFA) molecules the phospholipid membrane platforms are derived from the endoplasmic reticulum (activated and derived from RORgamma then from ROR-beta functions) and are rich in (at least) cholesterol. It has been observed that the phospholipase A2 (PLA2) enzyme family is activated in WNVKUN-infected [29]. 
During inflammation and infections the first effective replay key is ROR-gamma to act on inflammations to convert purines to pyrimidine for hydrophobic acids biosynthesis for contributing the beta-oxidation processes, then the ROR-beta activities for producing active linear beta-subunits and active linear beta-genes and linear autophagy for acting on inflammations molecules (including branched fatty acids, and carbohydrate), then the effect of phospholipase will be followed the beta-effects for the phospholipid biosynthesis and for converting the linear subunits and linear active genes into twisted oval rounded shape for acquire phospholipid protections for their internal components and create the required cells biological shapes and their inner cells granules protected by their phospholipid membranes including nucleus and ribosomes.

So, the function of ROR-alpha (fatty-acyl-CoA-phospholipase) is to convert the inelastic long open thread protein into a flexible oval rounded cell shaped through the formation of the phospholipid membranes to involve its own inner cells granules during cells proliferations including nucleus, ribosomes, lysosome, and peroxisomes which will act as necessary anti-inflammatory effects during the effective pathological periods.

During the pathological anti-inflammatory effects the phospholipid membranes will be analyzed due to active linear beta-subunits, Cox2 and ATPase that will release the prostaglandins PG1, PG2, PG3 upon phospholipid membranes hydrolysis for releasing their effective active units contents on inflammation molecules that will help the TNF-a and TXA2 beta-subunits synthesis which will strengthen immune and will resynthesis the cells shape again by reforming phospholipid membranes again during cells proliferations.

The same processes will occur for sestrin molecules biosynthesis, that sestrin-Leu-1 synthesis will need firstly the activities of ROR-gamma for resynthesis the Leu and hydrophobic acids for joining and bonding to sestrin molecules for completing full effective molecular structure for Sestrins-Leu molecules which can be synthesis in liver where the ROR-gamma is so necessary for liver cells full function and activities.

\section{Conclusion}

The effects of ATPase and COX-2 enzymes start firstly on pronutrients-mTOR molecules in protein, lipid, and carbohydrate biosynthesis for releasing the long fatty acids molecules, then secondly the mitochondrial effects on the long fatty acids chains for producing the three RORs active isoforms which are considered as are three active enzymatic acyl-CoA each is containing one of mitochondrial enzymes : 1, enzymatic acyl-CoA-phospholipase (ROR-alpha) which has the imp roles in anti-inflammatory function and in phospholipid synthesis, 2, acyl-CoA-synthase (ROR-beta) which has the beta oxidative biosynthesis for TNF-a and TXA2 subunits synthesis, 3, acyl-CoA-synthetase (ROR-gamma) which has the function of carbohydrate biosynthesis where maintain the proper balance of purines related to pyrimidine in blood tissues and has the necessary roles for hydrophobic amino acids and fatty acids biosynthesis.

Peroxisomal organelles are made basically by mitochondrial effect on fatty acids due to RORs genes transcriptional filter and consist of the three RORs isoforms receptors alpha, beta, \& gamma) for easier fastest processes for alpha beta, and gamma oxidations.

The beta oxidative process occur due to the activity of synthase for running the TNF-a and TXA2 biosynthesis for increasing immune efficiency, alpha oxidative processes is the fatty acyl-CoA phospholipase activities for lipoprotein and phospholipid biosynthesis where that alpha and beta oxidations are linked and contributing together for running their activities and producing $\mathrm{H} 2 \mathrm{O}$ from the peroxisomal processes, but gamma oxidative processes are linked to type of nutrients mTOR molecules, and to availabilities of Ser amino acids for releasing Thymine kinases proteins for the acyl-CoA synthetase biosynthesis, and linked to Thymine pyrimidine and production from purines nucleotides during carbohydrate metabolism.

At the time of decreasing or inhibition in acyl-CoA phospholipase and in gamma acyl-CoA synthetase will lead to accumulation of hydrogen peroxide and downstream of reactive oxygen species.

\section{Conflict of Interest}

The Author declare that the research work has been conducted in the absence of any commercial or financial relationships, that could be construed as a potential conflict of interest.

\section{References}

1. Trisha J Grevengoed, Eric L Klett, Rosalind A Coleman (2014) AcylCoA Metabolism and Partitioning. Annu Rev Nutr 34: 1-30.

2. Rennison JH, McElfresh TA, Okere IC, Patel HV, Foster AB, et al. (2008) Enhanced acyl-CoA dehydrogenase activity is associated with improved mitochondrial and contractile function in heart failure. Cardiovasc Res 79(2): 331-340

3. Abo Alrob O, Lopaschuk GD (2014) Role of CoA and acetyl-CoA in regulating cardiac fatty acid and glucose oxidation. Biochem Soc Trans 42(4): 1043-1051.

4. Russell A DeBose Boyd (2008) Feedback regulation of cholesterol synthesis: sterol-accelerated ubiquitination and degradation of HMG CoA reductase. Cell Research 18: 609-621.

5. Matt TBianchi(2014) Chapter 5 -Essentials of Sleep Neuropharmacology. Therapy in Sleep Medicine p. 62-82.

6. Dimitris Tousoulis (2018) Coronary Artery Disease from Biology to Clinical Practice. pp. 237-271.

7. Rongtao Zhu, Zhibing Ou, Xiongzhong Ruan, Jianping Gong (2012) Role of liver X receptors in cholesterol efflux and inflammatory signaling (Review) . Molecular Medicine Reports 5(4): 895-900.

8. Qi Shen, Yuchen Bai, Ken C N Chang, Yongjun Wang, Thomas P Burris, et al. (2011) Liver X Receptor-Retinoid X Receptor (LXR-RXR) Heterodimer Cistrome Reveals Coordination of LXR and AP1 Signaling in Keratinocytes. J Biol Chem 286(16): 14554-14563.

9. Jalil Antoine, Bourgeois Thibaut, Ménégaut Louise, Lagrost Laurent, Thomas Charles, et al. (2019) Revisiting the Role of LXRs in PUFA Metabolism and Phospholipid Homeostasis. International Journal of Molecular Sciences 20(15): 3787.

10. Thomas A Lagace (2016) Phosphatidylcholine: Greasing the Cholesterol Transport Machinery. Lipids Insights 8(S1): 65-73.

11. Allegretti Chiara, Denuccio Francesca, Rossato Letizia, D’Arrigo Paola (2020) Polar Head Modified Phospholipids by Phospholipase D-Catalyzed Transformations of Natural Phosphatidylcholine for Targeted Applications: An Overview. Catalysts 10(9): 997.

12. Gabriela Andrejeva, Sharon Gowan, Gigin Lin, Anne Christine LF Wong, Elham Shamsaei, et al. (2019) De novo phosphatidylcholine synthesis is required for autophagosome membrane formation and maintenance during autophagy. Autophagy 16(6): 1044-1060.

13. A Yamashita, N Kawagishi, T Miyashita, T Nagatsuka, T Sugiura (2001) ATP-independent Fatty Acyl-Coenzyme A Synthesis from Phospholipid coenzyme a-dependent transacylation activity toward lysophosphatidic acid catalyzed by acyl-coenzyme a:lysophosphatidic acid acyltransferase. J Biol Chem 276(29): 26745-26752.

14. Li R, Maminishkis A, Banzon T, Wan Q, Jalickee S, et al. (2009) IFN \{gamma\} regulates retinal pigment epithelial fluid transport. Am J Physiol Cell Physiol 297(6): C1452-1465.

15. Rong R, Ahn JY, Chen P, Suh PG, Ye K (2003) Phospholipase activity of phospholipase $\mathrm{C}$-gammal is required for nerve growth factor-regulated MAP kinase signaling cascade in PC12 cells. Journal of Biological Chemistry 278(52): 52497-52503.

16. Christine Crumbley, Yongjun Wang, Subhashis Banerjee, Thomas P. Burris (2012) Regulation of Expression of Citrate Synthase by the 
Retinoic Acid Receptor-Related Orphan Receptor a (RORa) PLoS One 7(4): e33804.

17. Yan Wang, Jernej Godec, Khadija Ben-Aissa, Kairong Cui, Keji Zhao, et al. (2014) The transcription factors T-bet and Runx are required for the ontogeny of pathogenic interferon- $\gamma$-producing $\mathrm{T}$ helper 17 cells Immunity 40(3): 355-366.

18. Shujiong Feng, Song Xu, Zhenzhen Wen, Yongliang Zhu (2015) Retinoic acid-related orphan receptor ROR $\beta$, circadian rhythm abnormalities and tumorigenesis (Review). International Journal of Molecular Medicine 35(6): 1493-1500.

19. Fátima Camões, Markus Islinger, Sofia C Guimarães, Sreedhar Kilaru, Martin Schuster (2015) New insights into the peroxisomal protein inventory: Acyl-CoA oxidases and-dehydrogenases are an ancient feature of peroxisomes. Biochimica et Biophysica Acta (BBA) Molecular Cell Research 1853(1): 111-125.

20. Ashraf Marzouk El Tantawi (2021) Pyrimidine within Tyrosine kinase and other (PSTGk, PSTCk, PSTAk) kinases produced from mTOR-FOX binding through effects of Ser/Thr phosphorylation mechanism play necessary rules for removing insulin resistance, and tumors. Organic Chemistry: Current Research Journal 10(4): 1-10.

21. Stanley R Terlecky, Jay I Koepke, Paul A Walton (2006) Peroxisomes and Aging. Biochimica et Biophysica Acta (BBA) - Molecular Cell Research 1763(12): 1749-1754.

22. Fátima Camões, Markus Islinger, Sofia C Guimarães, Sreedhar Kilaru, Martin Schuster, et al. New insights into the peroxisomal protein inventory: Acyl-CoA oxidases and-dehydrogenases are an ancient feature of peroxisomes. Biochimica et Biophysica Acta (BBA) Molecular Cell Research 1853(1): 111-125.

23. Paul A Watkins, Jessica M Ellis (2012) Peroxisomal acyl-CoA synthetases. Biochimica et Biophysica Acta (BBA) - Molecular Basis of Disease 1822(9): 1411-1420.

24. Barbara Lisowska, Dariusz Kosson, and Karolina Domaracka (2018) Lights and shadows of NSAIDs in bone healing: the role of prostaglandins in bone metabolism. Des Devel Ther 2018: 1753-1758.

25. Ramgolam VS, Sha Y, Jin J, Zhang X, Markovic-Plese S (2009) IFN-beta inhibits human Th17 cell differentiation. J Immunol 183(8): 5418-5427.

26. Jon M Evasovic, Cherie A Singer (2019) Regulation of IL-17A and implications for TGF- $\beta 1$ comodulation of airway smooth muscle remodeling in severe asthma. Lung Cellular and Molecular Physiology 316(5): 843-868.

27. Daniel A Giles, Maria E Moreno-Fernandez, Traci E Stankiewicz, Monica Cappelletti, Stacey S Huppert, et al. (2016) Regulation of Inflammation by IL-17A and IL-17F Modulates Non-Alcoholic Fatty Liver Disease Pathogenesis. PLoS ONE 11(2):e0149783.

28. Yangdongling Liu, Ariana Gray Bé, Victor W Or, Michael R Alves, Vicki $\mathrm{H}$ Grassian, et al. (2020) Challenges and Opportunities in MolecularLevel Indoor Surface Chemistry and Physics. Cell Reports Physical Science 1(11)

29. Ines Teichert (2018) Adenosine to inosine mRNA editing in fungi and how it may relate to fungal pathogenesis. PLOS Pathogens 14(4): e1007029. 